\title{
Erratum
}

\section{Erratum to "Interleukin 4 haplotypes of susceptibility to chronic periodontitis are associated with IL-4 protein levels but not with clinical outcomes of periodontal therapy" [Hum. Immunol. 74 (2013) 1688-1695]}

\author{
Giovana Anovazzi a,b, Livia S. Finoti ${ }^{\text {a,b }}$, Sâmia C.T. Corbi ${ }^{\mathrm{a}, \mathrm{b}}$, Yeon J. Kim ${ }^{\mathrm{b}}$, Andrea M. Marcaccini ${ }^{\mathrm{c}}$, \\ Raquel F. Gerlach ${ }^{c}$, Marisa V. Capela ${ }^{d}$, Silvana R.P. Orrico ${ }^{b}$, Joni A. Cirelli ${ }^{b}$, Raquel M. Scarel-Caminaga ${ }^{a, *}$ \\ ${ }^{a}$ Department of Morphology, School of Dentistry at Araraquara, UNESP - São Paulo State University, Araraquara, São Paulo, Brazil \\ ${ }^{\mathrm{b}}$ Department of Oral Diagnosis and Surgery, School of Dentistry at Araraquara, UNESP - São Paulo State University, Araraquara, São Paulo, Brazil \\ ${ }^{c}$ Department of Morphology, Stomatology and Physiology, Dental School of Ribeirao Preto, USP - University of São Paulo, Ribeirao Preto, São Paulo, Brazil \\ ${ }^{\mathrm{d}}$ Department of Physical Chemistry, Institute of Chemistry at Araraquara, UNESP - São Paulo State University, Araraquara, São Paulo, Brazil
}

The author has discovered errors in Table 3 of their article. The corrected Table 3 is provided below.

Table 3

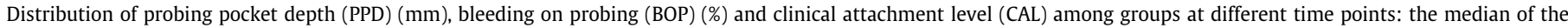
measurements (min-max).

\begin{tabular}{|c|c|c|c|c|c|}
\hline & Groups & $\begin{array}{l}\text { Baseline } \\
\text { Median (min-max) }\end{array}$ & $\begin{array}{l}45 \text { days } \\
\text { Median (min-max) }\end{array}$ & $\begin{array}{l}90 \text { days } \\
\text { Median (min-max) }\end{array}$ & $\begin{array}{l}\text { Difference among } \\
\text { the periods }{ }^{I I}\end{array}$ \\
\hline \multirow[t]{6}{*}{$\mathrm{PPD}(\mathrm{mm})$} & SCP & $2.4(2.0-3.9)$ & $1.9(1.2-3.8)$ & $1.8(1.3-3.7)$ & $<0.0001$ \\
\hline & $\mathrm{SH}$ & $1.6(1.1-2.1)^{*}$ & $1.5(1.2-1.9)^{*}$ & $1.2(1.0-1.9)^{*}$ & NS \\
\hline & PCP & $2.3(2.1-3.7)$ & $1.6(1.5-2.3)$ & $1.3(1.2-1.5)$ & 0.0001 \\
\hline & $\mathrm{PH}$ & $1.5(1.1-1.9)^{\dagger}$ & $1.2(1.0-1.6)^{\dagger}$ & $1.1(1.0-1.3)^{\dagger}$ & NS \\
\hline & Difference between the groups ${ }^{*}$ & $0.00005^{*}$ & $0.004^{*}$ & $0.002^{*}$ & \\
\hline & & $0.000001^{\dagger}$ & $0.0003^{\dagger}$ & $0.004^{\dagger}$ & \\
\hline \multirow[t]{6}{*}{ BOP $(\%)$} & SCP & $26.9(11.3-70.1)$ & $6.8(2.9-18.7)$ & $3.6(0.6-10.5)$ & $<0.0001$ \\
\hline & $\mathrm{SH}$ & $0.61(0-14.8)^{*}$ & $0.3(0-6.0)^{*}$ & $0.003(0-3.0)^{*}$ & 0.007 \\
\hline & PCP & $16.0(7.5-54.8)$ & $4.0(2.4-8.0)$ & $2.5(0.1-5.0)$ & 0.001 \\
\hline & $\mathrm{PH}$ & $0.59(0-13.0)^{\dagger}$ & $0,5(0-3.7)^{\dagger}$ & $0.002(0-1.2)^{\dagger}$ & $<0.0001$ \\
\hline & Difference between the groups ${ }^{*}$ & $0.00003^{*}$ & $0.00008^{*}$ & $0.0016^{*}$ & \\
\hline & & $0.00013^{\dagger}$ & $0.0027^{\dagger}$ & $0.00065^{\dagger}$ & \\
\hline \multirow[t]{3}{*}{$\mathrm{CAL}(\mathrm{mm})$} & SCP & $2.7(2.1-4.2)$ & $2.2(1.5-3.8)$ & $1.8(1.3-3.7)$ & 0.008 \\
\hline & PCP & $2.8(2.1-5.2)$ & $2.2(1.5-2.8)$ & $1.6(1.3-3.1)$ & 0.001 \\
\hline & Difference between the groups ${ }^{\S}$ & NS & NS & NS & \\
\hline \multirow{3}{*}{$\mathrm{PPD} \geqslant 5 \mathrm{~mm}$ and $\mathrm{CAL} \geqslant 3 \mathrm{~mm}$ (\% sites) } & SCP & $12.0(3.0-37.0)$ & $4.0(1.0-13.0)$ & $2.0(0-7.0)$ & 0.0001 \\
\hline & PCP & $13.0(4.0-29.0)$ & $4.0(1.0-10.0)$ & $1.0(0-3.0)$ & 0.0001 \\
\hline & Difference between the groups ${ }^{\S}$ & NS & NS & NS & \\
\hline
\end{tabular}

\footnotetext{
* Significant differences compared with the group SCP the same period.

† Differences when compared to group PCP the same period. NS: not significant.

$\S$ Mann-Whitney test for the evaluations between the groups in the same period.

II Friedman test for evaluation between different periods.
}

DOI of original article: http://dx.doi.org/10.1016/j.humimm.2013.08.286

* Corresponding author. Address: Department of Morphology, School of Dentistry at Araraquara, UNESP - São Paulo State University, CP. 331, CEP 14801-903 Araraquara, São Paulo, Brazil.

E-mail address: raquel@foar.unesp.br (R.M. Scarel-Caminaga). 